\section{ENIAC 2013 Special Issue}

\author{
Gustavo E. A. P. A. Batista - Myriam Delgado • \\ Flávia Bernardini
}

Published online: 3 September 2015

(C) Springer Science+Business Media Dordrecht 2015

The 2013 National Meeting of Artificial and Computational Intelligence (ENIAC 2013) was the tenth in a series of successful symposia in the area of Artificial Intelligence. Since its first edition in 1997, ENIAC has offered a broad forum for theoretical research and applications of Artificial and Computational Intelligence, thus allowing the exchange of experience between researchers, practitioners, educators and students of these areas.

Among the 142 papers published in ENIAC 2013, the conference program committee and program chairs identified the 9 best-evaluated papers. The authors of the selected papers were invited to submit expanded versions of their conference papers to this special issue. All expanded papers were reviewed by at least two additional international reviewers and at the end of the reviewing process, 7 papers were accepted for publication. In what follows, we briefly summarize the contents of this special issue papers.

G. E. A. P. A. Batista ( $\square)$

Universidade de São Paulo, São Paulo, Brazil

e-mail: gbatista@icmc.usp.br

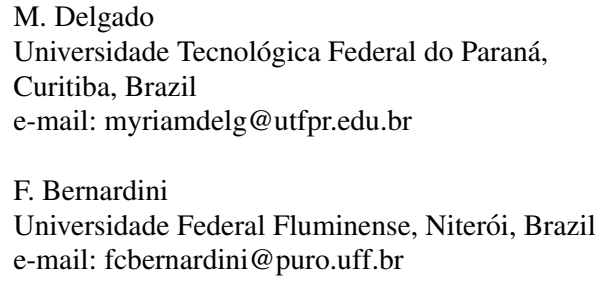

Luis Lorena, André Carvalho and Ana Lorena. Filter Feature Selection for One-Class Classification. Feature selection is an important and very active area of research. Reducing the number of features can reduce the computational resources in learning time and benefit some algorithms that are sensible to irrelevant or redundant features. In this paper, the authors endeavor in the challenging problem of feature selection for one-class classification problems. Their filter approach uses ranking composition methods to aggregate the scores of five feature selection methods adapted to the one-class context. The idea is that each measure provides a different view of the data and the ranking composition method will integrate these views in a single final feature set. The results obtained in 30 one-class datasets indicate a significant reduction in number of features while most of the classification performance is retained.

Túlio Alberto, Johannes Lochter and Tiago Almeida. Post or Block? Advances in Automatically Filtering Undesired Comments. In this paper the authors evaluate the use of machine learning techniques in the identification of a special form of spam, undesired comments in websites such as blogs and news. Detecting undesired comments is significantly more difficult than traditional spam filtering. These comments are usually short and impregnated with slang, acronyms and misspelled words. Additionally, real people instead of automatic bots usually enter comment spam. The paper provides a comprehensible evaluation with 15 classifiers/settings and 4 
evaluation measures. They conclude that support vector machine, logistic regression and stacking ensemble methods provide the best results when trained with attributes extracted from the text messages and posting information.

Everton Cherman, Newton Spolaôr, Jorge Valverde-Rebaza and Maria Carolina Monard. Lazy Multi-label Learning Algorithms Based on Mutuality Strategies. In this paper, the authors investigate the problem of multi-label classification, in which an example can be associated to one or more labels. They propose two strategies, MLMUT and MLnotMUT, to find a set of examples that will vote for the labels in a $k$-nearest neighbor multi-label approach. The results show that the proposed methods generally outperform the methods based on transformation approach and outperform MLkNN, a state-of-the-art algorithm for multi-label learning, when the F-Measure is considered.

Paulo Pisani, Ana Lorena and André Carvalho. Adaptive Positive Selection for Keystroke Dynamics. In this paper, the authors frame the problem of user authentication using biometric features in a data stream context. The idea is that biometric features evolve over time. For instance, keystroke dynamics, which recognizes the user by typing rhythm, may change as the user becomes more familiarized with the password. The authors use machine learning techniques, in particular immune positive selection algorithms to adapt to the concept drifts in the absence of true labels. The results show that the proposed method can improve false rejection rates.

Gabriela Biondi and Ronaldo Prati. Setting Parameters for Support Vector Machines using Transfer Learning. The paper proposes a transfer learning approach for setting the parameters of support vector machine classifiers. Support vector machines can be very sensitive to their free parameters. The community usually uses search procedures in the training set to find appropriate values for the parameters. However, it is quite time consuming process, especially if you have several datasets. The authors propose to use data complexity measures to characterize different datasets. Given a dataset at hand, they look for the most similar in terms of the complexity measures and transfer the best parameter setting to the new dataset. A comprehensive evaluation with 26 benchmark datasets shows that the proposed approach is very competitive to the popular grid search.

Diego Silva, Vinícius Souza, Daniel Ellis, Eamonn Keogh and Gustavo Batista. Exploring Low Cost Laser Sensors to Identify Flying Insect Species. The paper describes an application of machine learning techniques to automatically recognize flying insect species. The authors propose an inexpensive laser sensor that captures wing movement data. The paper main contribution is a broad evaluation of signal processing techniques to identify relevant features and machine learning systems to induce classifiers over the extracted features. The results show that the classifiers can achieve approximately $90 \%$ accuracy rate for 9 insect species and $95 \%$ for a binary classifier that recognizes disease vector mosquitoes.

Heider Berlink, Nelson Kagan, Anna Costa. Intelligent Decision-Making for Smart Home Energy Management. Smart grids is changing the way we consume energy by allowing consumers to generate energy in their houses and possibly injecting the surplus in the market. In this paper, the authors investigate the use of intelligent decision-making support systems to identify price tendencies in the energy market and help the user to decide to sell or store the surplus energy. The paper proposes an approach based on reinforcement learning. The results show a significant increase in long-term profit when the proposed approach is used compared to a naïve greedy policy considering the North American and Brazilian energy markets.

This special issue would not have been possible without the help of many people. We would like to especially thank the additional reviewers for their hard work for timely reviewing the papers of the special issue. We also would like to thank the editor-in-chief of the Journal of Intelligent \& Robotics Systems, Dr. Kimon P. Valavanis, for supporting this special issue since its initial proposal and Marie Veth Chua, from the Editorial Office, for promptly answering our innumerous questions. 